\title{
Regular magnetic black holes and monopoles from nonlinear electrodynamics
}

\author{
K.A. Bronnikov円 \\ Centre for Gravitation and Fundam. Metrology, VNIIMS, 3-1 M. Ulyanovoy St., Moscow 117313, Russia; \\ Institute of Gravitation and Cosmology, PFUR, 6 Miklukho-Maklaya St., Moscow 117198, Russia
}

\begin{abstract}
It is shown that general relativity coupled to nonlinear electrodynamics (NED) with the Lagrangian $L(F), F=$ $F_{\mu \nu} F^{\mu \nu}$ having a correct weak field limit, leads to nontrivial spherically symmetric solutions with a globally regular metric if and only if the electric charge is zero and $L(F)$ tends to a finite limit as $F \rightarrow \infty$. Properties and examples of such solutions, which include magnetic black holes and solitonlike objects (monopoles), are discussed. Magnetic solutions are compared with their electric counterparts. A duality between solutions of different theories specified in two alternative formulations of NED (called the FP duality) is used as a tool for this comparison.
\end{abstract}

PACS: 04.20.Jb, 04.20.Dw, 04.70.Bw

\section{Introduction}

General relativity, despite its nonlinearity, is apparently lacking an effective self-restriction mechanism, and the existence of singularities seems to be its inevitable, though undesired, feature. Reasonable, regular solutions for macroscopic bodies such as stars are obtained with matter whose pressure opposes gravity, whereas microobjects, extreme states of matter and/or strong gravitational fields probably need a purely field description.

The choice of a field source able to do the job is a separate task, and, in particular, for spherically symmetric configurations there are quite a number of nonexistence theorems [1]. Non-Abelian gauge fields yield regular black hole solutions [2], but they are known only numerically. The regular black hole solution of Ref. [3] with a de Sitter core is expressed in terms of pressure and density rather than fields. An especially attractive class of field theories for seeking regular models is nonlinear electrodynamics (NED) with gauge-invariant Lagrangians $L(F)$, $F=F_{\mu \nu} F^{\mu \nu}$, since its energy-momentum tensor (EMT) $T_{\mu}^{\nu}$ has the symmetry $T_{0}^{0}=T_{1}^{1}$ and is thus insensitive to boosts in the radial direction, which is a property of vacuum 3, 1. Such theories, in particular, the Born-Infeld NED, recently gained much attention as limiting cases of certain models of string theory (see [5] for reviews). It has been shown, however [6, 7], that in NED with any $L(F)$ such that $L \sim F$ at small $F$ (the Maxwell weak-field limit), static, spherically symmetric generalrelativistic configurations with a nonzero electric charge cannot have a regular center. As will be shown below, the same is true for dyonic configurations, where both electric and magnetic charges are present; regular solutions with wormhole topology also cannot exist for this system.

The prohibition does not concern purely magnetic solutions, and, quite surprisingly, there is a whole class of

\footnotetext{
${ }^{1}$ e-mail: kb@rgs.mccme.ru
}

regular solutions with a nonzero magnetic charge. Regularity is here understood in a physical sense: although $F$ is infinite at the center, the EMT is finite and the metric is regular (at least $C^{2}$ ) while the forces applied to test particles are finite everywhere and vanish at the center. The main aim of this paper is to present and to discuss these solutions. We will also compare them with their electric analogs, in particular, with the solutions found by Ayón-Beato and García [8-10]. For this comparison we use a duality between spherically symmetric solutions of different NED specified in two alternative $(F$ and $P$ ) frameworks: the original, Lagrangian framework and another one, obtained from it by a Legendre transformation [11.

Throughout the paper all relevant functions are assumed to be sufficiently smooth, unless otherwise indicated.

\section{Nonexistence theorems}

Let us begin with a proof of two simple nonexistence theorems extending the results of [6, 7].

Consider NED in general relativity, with the action

$$
S=\frac{1}{16 \pi} \int d^{4} x \sqrt{-g}[R-L(F)], \quad F \stackrel{\text { def }}{=} F_{\mu \nu} F^{\mu \nu},
$$

where $R$ is the scalar curvature, $F_{\mu \nu}=\partial_{\mu} A_{\nu}-\partial_{\nu} A_{\mu}$ is the electromagnetic field, and $L$ is an arbitrary function leading to the Maxwell theory at small $F: L(F) \approx F$ as $F \rightarrow 0$. The tensor $F_{\mu \nu}$ obeys the dynamic equations and the Bianchi identities,

$$
\nabla_{\mu}\left(L_{F} F^{\mu \nu}\right)=0, \quad \nabla_{\mu}^{*} F^{\mu \nu}=0,
$$

where an asterisk denotes the Hodge dual and $L_{F}=$ $d L / d F$. 
A static, spherically symmetric metric can be written in the general form

$$
d s^{2}=\mathrm{e}^{2 \gamma(u)} d t^{2}-\mathrm{e}^{2 \alpha(u)} d u^{2}-r^{2}(u) d \Omega^{2}
$$

where $d \Omega^{2}=d \theta^{2}+\sin ^{2} \theta d \phi^{2}$ and $u$ is a radial coordinate. A regular center, by definition, takes place where $r=0$, if all algebraic curvature invariants are finite there and, in addition, there is a correct limiting value of the circumference to radius ratio (local flatness at the center, the absence of a conical singularity). If one chooses the curvature coordinates, $u=r$, the local flatness condition reads $\mathrm{e}^{\alpha(0)}=1$.

The tensor $F_{\mu \nu}$ compatible with spherical symmetry can involve only a radial electric field $F_{01}=-F_{10}$ and a radial magnetic field $F_{23}=-F_{32}$. Eqs. (2) give

$$
r^{2} \mathrm{e}^{\alpha+\gamma} L_{F} F^{01}=q_{\mathrm{e}}, \quad F_{23}=q_{\mathrm{m}} \sin \theta
$$

where $q_{\mathrm{e}}$ and $q_{\mathrm{m}}$ are the electric and magnetic charges, respectively. As follows from (画),

$$
\begin{aligned}
f_{\mathrm{e}} \stackrel{\text { def }}{=} 2 F_{01} F^{10} & =2 q_{\mathrm{e}}^{2} L_{F}^{-2} r^{-4} \geq 0, \\
f_{\mathrm{m}} \stackrel{\text { def }}{=} 2 F_{23} F^{23} & =2 q_{\mathrm{m}}^{2} r^{-4} \geq 0,
\end{aligned}
$$

and the Einstein equations may be written in the form

$$
\begin{aligned}
& -G_{\mu}^{\nu}=T_{\mu}^{\nu}=-2 L_{F} F_{\mu \alpha} F^{\nu \alpha}+\frac{1}{2} \delta_{\mu}^{\nu} L \\
& =\frac{1}{2} \operatorname{diag}\left(L+2 f_{\mathrm{e}} L_{F}, L+2 f_{\mathrm{e}} L_{f}, L-2 f_{\mathrm{m}} L_{F}, L-2 f_{\mathrm{m}} L_{F}\right)
\end{aligned}
$$

Theorem 1. The field system (1), with $L(F)$ having a Maxwell asymptotic $\left(L \rightarrow 0, L_{F} \rightarrow 1\right.$ as $\left.F \rightarrow 0\right)$, does not admit a static, spherically symmetric solution with a regular center and a nonzero electric charge.

Proof. Since the Ricci tensor for the metric (3) is diagonal, the invariant $R_{\mu \nu} R^{\mu \nu} \equiv R_{\mu}^{\nu} R_{\nu}^{\mu}$ is a sum of squares, hence each component $R_{\mu}^{\mu}$ (no summing) is finite at a regular space-time point. Then each component of the EMT $T_{\mu}^{\nu}$ is finite as well, hence, as follows from (7),

$$
\left(f_{\mathrm{e}}+f_{\mathrm{m}}\right)\left|L_{F}\right|<\infty .
$$

Suppose first that $q_{\mathrm{m}}=0, q_{\mathrm{e}} \neq 0$ and thus $f_{\mathrm{m}}=0$ and $F=-f_{\mathrm{e}}$. Therefore, by (5) and (9), at a regular center $F L_{F}$ is finite whereas $F L_{F}^{2} \rightarrow \infty$. Combined, these conditions lead to $F \rightarrow 0$ and $L_{F} \rightarrow \infty$, that is, a strongly non-Maxwell behavior at small $F$. Thus for purely electric fields the theorem is valid.

Suppose now $q_{\mathrm{e}} \neq 0$ and $q_{\mathrm{m}} \neq 0$. Then (9) should hold for $f_{\mathrm{e}}$ and $f_{\mathrm{m}}$ taken separately. As stated previously, this condition applied to $f_{\mathrm{e}}$ combined with (5) leads to $L_{F} \rightarrow \infty$. But $f_{\mathrm{m}}$ also tends to infinity as $r \rightarrow 0$, so even stronger $f_{\mathrm{m}} L_{F} \rightarrow \infty$, violating (9). The theorem is proved.

A regular center is, however, not a necessary feature of a regular static, spherically symmetric space-time: there might be no center at all. Let us find out whether or not our system (11) can behave like this.

If a center is lacking, the metric function $r=\left(-g_{22}\right)^{1 / 2}$ is restricted below by some minimum value $r^{*}>0$. There are then two opportunities:

(i) $r(u)$ has one or several minima (as it happens, e.g., in a wormhole); $r$ itself then fails to be an admissible coordinate since it takes equal values at different spheres;

(ii) $r(u)$ monotonically approaches $r_{\min }$ as $u$ tends to a certain limiting value $u^{*}$; then the space-time can be nonsingular if it ends with a horn.

By definition, $a$ horn in a static, spherically symmetric space-time with the metric (1) is a region where, as $u$ tends to some value $u^{*}, r(u) \neq$ const and $g_{t t}=\mathrm{e}^{2 \gamma(u)}$ have finite limits while the integral $\ell=\int \mathrm{e}^{\alpha} d u$ diverges. In other words, a horn is an infinitely long (3-dimensional) "tube" of finite radius, with the clock rate remaining finite everywhere. It has an infinite spatial volume, and geodesics are infinitely continued along it as if in a wormhole throat of unlimited length. The condition $r \neq$ const discards purely cylindrical space-times, sometimes called flux tubes, which have no asymptotics. The definition of a horn follows the papers by Banks et al. [12], where "horned particles" were discussed as possible remnants of black hole evaporation.

The following theorem shows that these opportunities can never be realized if the EMT has the "vacuum" property $T_{0}^{0}=T_{1}^{1}$, and, in particular, for our system (1).

Theorem 2. Let the metric (3) obey the Einstein equations with an EMT satisfying the condition $T_{0}^{0}=T_{1}^{1}$. Then (i) the function $r(u)$ cannot have a regular minimum and (ii) the space-time cannot contain a horn.

Proof. Let us choose the $u$ coordinate by fixing the condition $\alpha=\ln r-\gamma$. The $\left(\begin{array}{l}0 \\ 0\end{array}\right)-\left(\begin{array}{l}1 \\ 1\end{array}\right)$ Einstein equation takes the form $d^{2}(\ln r) / d u^{2}=0$. Therefore

$$
r(u)=\mathrm{e}^{a u+b}, \quad a, b=\text { const },
$$

so that $r$ is either a constant (if $a=0$ ) or a strictly monotonic function. This proves item (i).

Suppose now that there is a horn. Then, by the above definition, $a \neq 0$, and a finite limit of $r$ in Eq. (10) as $u \rightarrow u^{*}$ means that $u^{*}$ is also finite. On the other hand, since $\int \mathrm{e}^{\alpha} d u$ diverges as $u \rightarrow u^{*}$, it follows that $\alpha \rightarrow$ $\infty$, which, by our coordinate condition, can only occur if $\gamma \rightarrow-\infty$, contrary to what was assumed. This completes the proof.

Some remarks are now in order. First, the absence of wormhole solutions can also be proved from the known fact that a static wormhole throat always implies a violation of the null energy condition 13 . This condition in our case reads $T_{0}^{0}-T_{1}^{1} \geq 0$ and is (marginally) observed 
by the identical zero at the left-hand side. Our proof is, however, more direct and explicit.

Second, the opportunity $\gamma \rightarrow-\infty$, mentioned in the proof of item (ii) of Theorem 2, generically corresponds to an event horizon. [It can happen in principle that $\mathrm{e}^{\gamma} \rightarrow 0$ at finite $r$ does not imply a horizon. Our system (11) with smooth $L(F)$ does not admit such cases, while horizons do exist, as can be seen from the solution below.]

Third, the above theorems do not contain an asymptotic flatness requirement, the proofs being of local nature. Therefore both theorems are readily extended to general relativity with a cosmological constant (the condition $T_{0}^{0}=T_{1}^{1}$ holds in its presence as well), where the spatial asymptotic can be de Sitter or anti-de Sitter.

\section{Regular magnetic solutions}

Both theorems were proved without entirely solving the Einstein equations. For our system (11), however, an exact solution can be obtained by quadratures in the general spherically symmetric case [6]. Indeed, the Maxwell-like equations are already integrated. Let us choose the curvature coordinates $u=r$, which is now safe since we know that $r$ has no extrema. Due to $T_{0}^{0}=T_{1}^{1}$, the corresponding Einstein equation gives $d(\alpha+\gamma) / d r=0 \Rightarrow \alpha+\gamma=0$ for a proper choice of the time unit. It remains to write the well-known relation for $\alpha(r)$ in terms of the energy density $T_{0}^{0}$, which follows from the $\left(\begin{array}{l}0 \\ 0\end{array}\right)$ Einstein equation:

$$
\begin{aligned}
\mathrm{e}^{2 \gamma}=\mathrm{e}^{-2 \alpha} & =A(r)=1-\frac{2 M(r)}{r}, \\
M(r) & =\frac{1}{2} \int T_{0}^{0}(r) r^{2} d r .
\end{aligned}
$$

Possible horizons occur at zeros of $A(r)$.

The only nontrivial case not covered by the theorems is $q_{\mathrm{e}}=0, q_{\mathrm{m}} \neq 0$, when there is still a hope to obtain a regular center. In this case the metric has the form (3) with (11) where

$$
M(r)=\frac{1}{4} \int L(F) r^{2} d r
$$

and $F=2 q_{\mathrm{m}}^{2} / r^{4}$. It is easily seen that a space-time with a regular center is indeed obtained for any $L(F)$ such that $L \rightarrow L_{\infty}<\infty$ as $F \rightarrow \infty$ when one integrates in Eq. (12) from 0 to $r$. Integration from 0 to $\infty$ then gives a unique mass $m=M(\infty)$ providing a regular center for given $q_{\mathrm{m}}$; hence the entire mass is of electromagnetic origin. A finite value of $M(\infty)$ is guaranteed by our assumption of a Maxwell asymptotic of $L(F)$ at small $F$ : as $r \rightarrow \infty$, $L \approx 2 q_{\mathrm{m}}^{2} / r^{4}$, and the integral (12) converges.

The EMT in (7) near $r=0$ takes the form $T_{\mu}^{\nu}=$ $\frac{1}{2} \delta_{\mu}^{\nu} L_{\infty}(1+o(1))$, and the metric is approximately de Sitter $\left(A(r)=1-\Lambda r^{2} / 3+o\left(r^{2}\right)\right)$, with the cosmological constant $\Lambda=L_{\infty} / 2$. The Riemann tensor at $r=0$ coincides with that of de Sitter space, so one need not explicitly calculate the curvature invariants to prove that the space-time is regular. The metric is at least $C^{2}$ smooth at $r=0$ but, depending on $L(F)$, may be even $C^{\infty}$ smooth, as will be seen in the example (29).

Suppose that $L(F)$ and the mass have been chosen as described. The space-time is then globally regular and can include horizons corresponding to zeros of $A(r)$, whose number and character determine the global structure [note that $A(0)=A(\infty)=1$ ]. In the absence of zeros, there is a regular Dirac-type magnetic monopole solution. The occurence of two simple zeros leads to the conventional Reissner-Nordström black hole structure, with the singularity replaced by a regular center. The intermediate case of one double zero gives the extremal Reissner-Nordström structure. Models with more numerous horizons can be constructed as well.

Due to the above theorems, our magnetic black holes and monopoles are the only types of static, spherically symmetric solutions to (1) with a globally regular metric.

The only trouble is an infinite magnetic induction $B$ $\left(B^{2}=f_{\mathrm{m}} / 2=F / 2\right)$ at $r=0$, whereas the magnetic field intensity $H$, obtained as a generalized momentum from the Lagrangian, is well-behaved everywhere including the center: $H^{2}=f_{\mathrm{m}} L_{F}^{2} / 2 \rightarrow 0$ as $r \rightarrow 0$. To judge whether or not the center is regular from a physical viewpoint, one should estimate the force applied to a charged test particle moving in the field under consideration. This test charge may be electric or magnetic since both are admitted by our assumptions. In a consistent approach, the equations of motion for a test particle (as well as those for an extended body) in nonlinear field theory should follow from the field equations and may be deduced along the lines of Refs. [14, 15]. Namely, the 4 -force vector is found as the total momentum flow $\int T_{\mu \nu} n^{\mu} d S$ through a closed surface surrounding the particle, where $n^{\mu}$ is the unit normal to such a surface and $T_{\mu \nu}$ is the total EMT of the summed electromagnetic field of the background static configuration and the test particle itself. An estimate in a proper approximation, taking into account the weakness of the particle's field, shows that this force is finite everywhere and vanishes at $r=0$. Therefore, despite the divergent $B$, our magnetic solutions may be called globally regular.

\section{4. $\quad F P$ duality and electric solutions}

Let us consider for comparison the electric analogs of our magnetic solutions. This is of particular interest since recently Ayon-Beato and García [8-10] suggested some examples of such solutions, describing configurations with $q_{\mathrm{e}} \neq 0, q_{\mathrm{m}}=0$ and a regular center. The properties of these solutions evidently contradict Theorem 1, but they only seem to circumvent it since, as we shall see, any model like those of [8-10] needs different Lagrangians in different ranges of the radial coordinate and therefore fails to be a solution for a particular Lagrangian $L(F)$.

The solutions of $[8-10]$ were found using an alternative form of NED (to be called the $P$ framework), 
obtained from the original one (the $F$ framework) by a Legendre transformation: one introduces the tensor $P_{\mu \nu}=L_{F} F_{\mu \nu}$ with its invariant $P=P_{\mu \nu} P^{\mu \nu}$ and considers the Hamiltonian-like quantity $\mathcal{H}=2 F L_{F}-L$ as a function of $P$; the theory is then reformulated in terms of $P$ and is specified by $\mathcal{H}(P) 11$. One then has:

$$
L=2 P \mathcal{H}_{P}-\mathcal{H}, \quad L_{F} \mathcal{H}_{P}=1, \quad F=P \mathcal{H}_{P}^{2}
$$

with $\mathcal{H}_{P}=d \mathcal{H} / d P$. Eqs. (2) and the EMT (7) are rewritten in the form

$$
\begin{gathered}
\nabla_{\mu} P^{\mu \nu}=0, \quad \nabla_{\mu}\left(\mathcal{H}_{P}{ }^{*} F^{\mu \nu}\right)=0, \\
T_{\mu}^{\nu}=-\frac{1}{2} \operatorname{diag}\left(\mathcal{H}-2 p_{\mathrm{m}} \mathcal{H}_{P}, \mathcal{H}-2 p_{\mathrm{m}} \mathcal{H}_{P},\right. \\
\left.\mathcal{H}+2 p_{\mathrm{e}} \mathcal{H}_{P}, \mathcal{H}+2 p_{\mathrm{e}} \mathcal{H}_{P}\right)
\end{gathered}
$$

where, by (14), in the spherically symmetric case,

$$
\begin{aligned}
p_{\mathrm{e}} & =2 P_{01} P^{10}=2 q_{\mathrm{e}}^{2} / r^{4}, \\
p_{\mathrm{m}} & =2 P_{23} P^{23}=2 q_{\mathrm{m}}^{2} \mathcal{H}_{P}^{2} / r^{4} .
\end{aligned}
$$

Comparing Eqs. (2), (5)-(8) with (14)-(17), one sees that they coincide up to the substitutions

$$
\left\{g_{\mu \nu}, F_{\mu \nu}, F, L(F)\right\} \longleftrightarrow\left\{g_{\mu \nu},{ }^{*} P_{\mu \nu},-P,-\mathcal{H}(P)\right\} .
$$

In other words, there is a duality between spherically symmetric solutions written in the $F$ and $P$ frameworks: any solution for a given Lagrangian $L(F)$, characterized by a certain metric function $A(r)$ and the field components $F_{01}$ and $F_{23}$, has a counterpart with the same $A(r)$ but with $F$ substituted by $-P, L$ by $-\mathcal{H}, F_{01}$ by $P_{23}$ and $F_{23}$ by $P_{01}$, and conversely. The functional dependence $-\mathcal{H}(-P)$ in the dual solution is the same as $L(F)$ in the original solution, but the choice of the function $L(F)$ itself is not restricted.

(It should be stressed that this duality, to be called $F P$ duality, connects solutions of different theories: given $L(F)$, the functional dependence $\mathcal{H}(P)=2 F L_{F}-$ $L$ is in general quite different from $L(F)$, an evident exception being the Maxwell theory, where $L=F=$ $\mathcal{H}=P$ and the present duality turns into the conventional electric-magnetic duality. So the $F P$ duality has nothing to do with the electric-magnetic one studied in Refs. [11, 16], where the field equations of a specific theory were required to be duality invariant, and this condition selected a narrow class of Lagrangians.)

In particular, any regular magnetic solution obtained for given $L(F)$ has a purely electric counterpart with a similar (up to the sign) dependence $\mathcal{H}(P)$. The metric has the form (11) with

$$
M(r)=-\frac{1}{4} \int \mathcal{H}(P) r^{2} d r .
$$

Given $\mathcal{H}(P)$, one should substitute $P=-2 q_{\mathrm{e}}^{2} / r^{4}$. A regular center exists if and only if $\mathcal{H}$ has a finite limit as $P \rightarrow-\infty$, and a mass that provides regularity for given
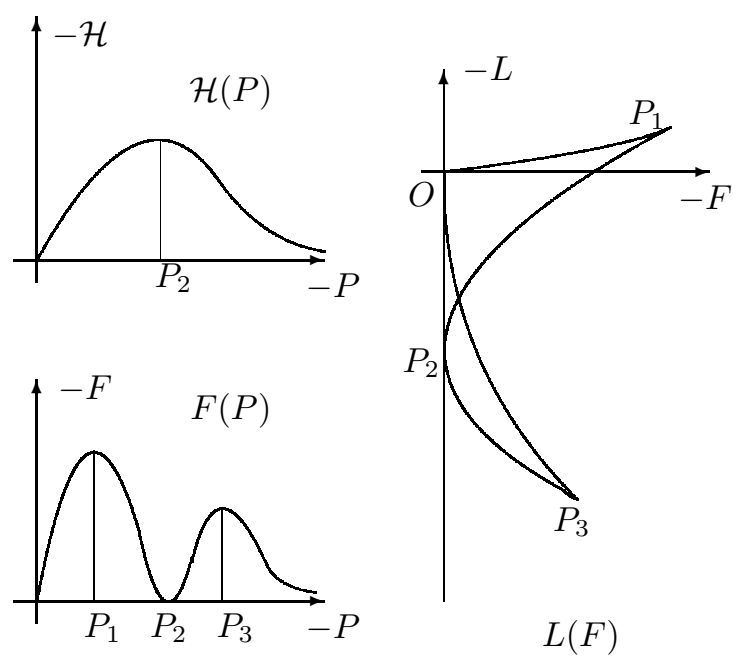

Figure 1: An example of qualitative behavior of $\mathcal{H}(P), F(P)$, and $L(F)$ in an electric solution

$q_{\mathrm{e}}$ is found by integration in (19) from 0 to $\infty$. This is how the regular solutions of [8-10] were obtained with the following choices of $\mathcal{H}(P)$ :

$$
\begin{aligned}
{[8]: \mathcal{H}(P) } & =P \frac{1-3 \Pi}{(1+\Pi)^{3}}+\frac{6}{q^{2} s}\left(\frac{\Pi}{1+\Pi}\right)^{5 / 2} \\
{[9]: \mathcal{H}(P) } & =P / \cosh ^{2}(s \sqrt{\Pi}) \\
\text { [10] } \mathcal{H}(P) & =P \frac{\exp (-s \sqrt{\Pi})}{(1+\Pi)^{5 / 2}}\left(1+\frac{3}{s} \sqrt{\Pi}+\Pi\right)
\end{aligned}
$$

where $\Pi=\sqrt{-q^{2} P / 2}$ and $s=|q| /(2 m), q=q_{\mathrm{e}}$ and $m$ being free parameters identified with the charge and mass of the configuration, respectively. The functions (20)-(22) behave like $P$ at small $P$, tend to finite limits as $P \rightarrow-\infty$ and thus lead to regular metrics.

Yet the $P$ framework is secondary: the Lagrangian dynamics is specified in the $F$ framework, and, since the $F \mapsto P$ transition is a mere substitution in the field equations, the two frameworks are only equivalent where the function $P(F)$ is monotonic. Recalling the proof of Theorem 1 for $q_{\mathrm{e}} \neq 0, q_{\mathrm{m}}=0$, one sees, however, that for any regular solution with a Reissner-Nordström asymptotic the function $F(P)=-f_{\mathrm{e}} \leq 0$ vanishes at both $r=0$ and $r \rightarrow \infty$. So it inevitably has at least one minimum at some $P=P^{*}<0$. It can be shown directly that at an extremum of $F(P)$ where $F=F^{*}<0$ the derivative $L_{F}$ has the same finite limit as $P \rightarrow P^{*}+0$ and $P \rightarrow P^{*}-0$, while $L_{F F}$ tends to infinities of opposite signs. Therefore the function $L(F)$ suffers branching, and its graph forms a cusp at $F=F^{*}$; different functions $L(F)$ correspond to $P>P^{*}$ and $P<P^{*}$.

Another kind of branching occurs at extrema of $\mathcal{H}(P)$, if any. There $F(P)$ behaves generically as $\left(P-P^{*}\right)^{2}$ while $L_{F} \rightarrow \infty$, and a graph of $L(F)$ smoothly touches 
the vertical axis $F=0$. The number of Lagrangians on the way from infinity to the center equals the number of monotonicity ranges of $F(P)$.

All this is readily seen for specific examples. A qualitative picture for the choice (21) is shown in Fig. 1.

In the simplest case when $\mathcal{H}(P)$ is monotonic (e.g., like $\tanh P), L(F)$ has only two branches $O P_{1}$ and $P_{1} P_{2}$, and $P_{2}$ already corresponds to $r=0$.

Thus any regular electric solution, being well-behaved with respect to the field equations in the $P$ framework, corresponds to different Lagrangians in different parts of space. This problem is absent for magnetic solutions since they are obtained directly in terms of $L(F)$.

\section{Comparison of effective metrics}

The troubles with the electric solutions concern only the properties of NED, while the metric is quite well-behaved. The same is true for the electric field $F_{01}$. However, termination of a theory with given $L(F)$ implies some violent electromagnetic phenomena. For their understanding let us consider the effective metric introduced by Novello et al. 17, 18.

$$
h^{\mu \nu}=g_{\mathrm{eff}}^{\mu \nu}=g^{\mu \nu} L_{F}-4 L_{F F} F^{\mu}{ }_{\alpha} F^{\alpha \nu} .
$$

As shown in [17, 18], NED photons propagate along null geodesics of this metric. For the space-time metric (11, with a purely electric field the effective metric reads

$$
\begin{aligned}
d s_{\mathrm{eff}}^{2} & =h_{\mu \nu} d x^{\mu} d x^{\nu}=\frac{1}{\Phi}\left[A(r) d t^{2}-\frac{d r^{2}}{A(r)}\right]-\frac{r^{2}}{L_{F}} d \Omega^{2} \\
\Phi & =L_{F}+2 F L_{F F}=\mathcal{H}_{P} / F_{P} .
\end{aligned}
$$

At an extremum $P=P^{*}$ of $F(P)$ where $F \neq 0$ (in particular, at the inevitable first minimum) one has $\Phi \rightarrow 0$ since $F_{P} \rightarrow 0$ while $\mathcal{H}_{P}$ is finite. This leads to a curvature singularity of the effective metric, at least if $P^{*}$ is not located on a horizon, $A \neq 0$. Another kind of singularity of the metric (24) accompanies possible extrema of $\mathcal{H}(P)$. All this is verified by calculating the Kretschmann scalar $K$. Even more importantly, according to 18, if a NED photon comes from an emitter at rest at point 1 to an observer at rest at point 2, the corresponding frequencies $f_{1}$ and $f_{2}$ are related by

$$
\frac{f_{2}}{f_{1}}=\frac{h_{t t}(1)}{\sqrt{g_{t t}(1)}} / \frac{h_{t t}(2)}{\sqrt{g_{t t}(2)}}=\frac{\Phi(2) / \sqrt{A(2)}}{\Phi(1) / \sqrt{A(1)}}
$$

where the second equality corresponds to the metric (24). If $\Phi(2)=\infty$ [as it happens at a termination point of $L(F)]$, then photons coming there are infinitely blueshifted and one may expect that they eventually lead to a real space-time singularity.

For a magnetic solution, instead of (24), we get

$$
d s_{\mathrm{eff}}^{2}=\frac{1}{L_{F}}\left[A(r) d t^{2}-\frac{d r^{2}}{A(r)}\right]-\frac{r^{2}}{\Phi} d \Omega^{2}
$$

where again $\Phi=L_{F}+2 F L_{F F}$. Instead of (25),

$$
\frac{f_{2}}{f_{1}}=\frac{L_{F}(2) / \sqrt{A(2)}}{L_{F}(1) / \sqrt{A(1)}} \text {. }
$$

At the center $(r=0, A=1)$ both $L_{F}$ and $\Phi$ vanish, the coefficient $h_{22} \rightarrow \infty$, i.e., behaves as if in a wormhole, whereas $h_{00} \rightarrow \infty$, which means that photons arriving there, if any, would be infinitely redshifted [see Eq. (27)]. Actually, photons cannot reach a place where $L_{F}=0$, as can be seen from an integral of their geodesic equation

$$
L_{F}^{-2} \dot{r}^{2}+\left[A(r) \Phi / r^{2}\right] l^{2}=\epsilon^{2}
$$

where the overdot is a derivative in the affine parameter, and $\epsilon$ and $l$ are the photon's constants of motion characterizing its initial energy and angular momentum. All curvature invariants of the metric (26) vanish at $r=0$. It is indeed a perfectly quiet place despite an infinite $F$.

Some peculiarities, however, occur on the way from infinity to the center. There is always a sphere $r=r^{*}$ on which $\Phi=0$. It can be seen as follows: $\Phi$ may be represented as $\Phi=2 \sqrt{F}\left(\sqrt{F} L_{F}\right)_{F}$. The quantity $\sqrt{F} L_{F}$ vanishes at both $r=0$ and $r=\infty$ and is nonzero between them, hence it has at least one extremum at $F \neq 0$ — this is where $\Phi=0$. The metric (26), due to blowingup of the coordinate spheres, has a singularity there, but the latter is actually unnoticed by NED photons, as is evident from Eq. (28). Generically $L_{F} \neq 0$ where $\Phi=0$, therefore the photon frequency also remains finite. The meaning of the very fact of a curvature singularity of the effective metric is yet to be understood.

If $L_{F}=0$ at some $F>0$, this also causes a singularity of Eq. (26), which acts as a potential wall (mirror) for NED photons, as is seen from (28). Accordingly, (27) shows that they are infinitely redshifted: $f_{2}$ vanishes if $L_{F}(2)=0$. No photons from outside can thus approach the center.

All this is in striking contrast to the picture obtained for an electric source - we now have potential walls instead of wells and redshifts instead of blueshifts.

\section{Example}

To have a specific example of a regular magnetic solution, let us employ the above $F P$ duality and consider, with slight modifications, the dependence (21), substituting $-\mathcal{H}$ with $L$ and $-P$ with $F$. An advantage of Eq. (21) [as well as (20) and (22)] is that it leads to a closed form of $M(r)$ and $A(r)$. Let us, however, slightly modify it, excluding an explicit dependence of $L$ on $m$ and $q$ : they should be integration constants, while $L$ may only contain fundamental constants or those originating from a deeper underlying theory. Moreover, to be able to describe systems with both electric and magnetic fields, where $F$ (and $P$ ) can have both signs, let us replace $-P$ with $|F|$ rather than $F$. So we put

$$
L(F)=F / \cosh ^{2}\left(a|F / 2|^{1 / 4}\right), \quad a=\text { const. }
$$


The use of $|F|$ violates analyticity of $L$ at $F=0$ : as required, $L_{F}(0)=1$, but $L_{F F}$ contains the discontinuous term $-a^{2}|2 F|^{-1 / 2} \operatorname{sign} F$. Though, in the range of interest, $F>0$, this $L(F)$ is well-behaved. Integration in Eq. (12) gives for a regular solution

$$
M(r)=\frac{|q|^{3 / 2}}{2 a}\left(1-\tanh \frac{a \sqrt{|q|}}{r}\right),
$$

so that $m=M(\infty)=|q|^{3 / 2} / 2 a\left(q=q_{\mathrm{m}}\right)$, and some relations from [9] are formally restored. In particular, the minimum value of $A(r)=1-2 M(r) / r$ [recall that $A(0)=1]$ depends on the ratio $\xi=m /|q|$, so that $A_{\text {min }}$ is negative for $\xi>\xi_{0} \approx 0.96$ (we deal with a black hole with two horizons), zero for $\xi=\xi_{0}$ (an extremal black hole with one double horizon) and positive for $\xi<\xi_{0}$ (a regular particle-like system). It is of interest that, given any specific value of the constant $a$ in Eq. (29), we can obtain all three types of solutions depending on the charge value: we have a non-extremal or extremal black hole if $|q| \leq 4 a^{2} / \xi_{0}^{2}$, or we have a particlelike solution (a monopole) otherwise. Despite the restriction imposed by the regularity condition, one finds all three types of regular solutions. This feature seems to be quite generic for proper nonlinear Lagrangians. One can also verify that the properties of the effective metric (26) confirm the above general observations.

One can also notice that, due to an exponential decay of $M(r)$ in Eq. (30), the metric is in this case $C^{\infty}$ smooth at $r=0$.

\section{Concluding remarks}

A more complete description of the properties of the present regular NED solutions, as well as others, requires a better understanding of the long-standing and non-trivial problem of motion of charged bodies in NED, probably following the lines of Refs. [14, 15, 19].

One more subject of interest for further study is the inclusion of another electromagnetic field invariant, ${ }^{*} F^{\mu \nu} F_{\mu \nu}$, into the Lagrangian in addition to $F$. This invariant is involved, in particular, in the Born-Infeld and Heisenberg-Euler NED Lagrangians; its appearance should be able to widen the diversity of regular black hole and monopole solutions. Related subjects are the FP duality between solutions of different theories involving both invariants and a possible extension of this duality to non-spherically symmetric configurations.

Acknowledgments. I acknowledge the partial financial support of the Russian Ministry of Science and Technologies and DFG Proj. 436 RUS 113/236/0(R) and the kind hospitality at Potsdam University. I am sincerely grateful to Irina Dymnikova, Yuri Rybakov, Vitaly Melnikov, Vladimir Dzhunushaliev, Gernot Neugebauer, Claus Lämmerzahl, Hans-Jürgen Schmidt and Martin Rainer for helpful discussions.

\section{References}

[1] K.A. Bronnikov and G.N. Shikin, "Self-gravitating particle models with classical fields and their stability". Series "Results of Science and Technology", Subseries "Classical Field Theory and Gravitation Theory", v.2, p. 4, VINITI, Moscow, 1991 (52 pp., in Russian)

[2] J.A. Smoller and A.G. Wassermann, Commun. Math. Phys. 151, 303 (1993);

P. Breitenlohner, P. Forgács and D. Maison, ibid., 163, 141 (1994)

[3] I. Dymnikova, Gen. Rel. Grav. 24, 235 (1992)

[4] I. Dymnikova, Phys. Lett. B 472, 33 (2000); gr-qc/9912116; gr-qc/0010016

[5] N. Seiberg and E. Witten, "String theory and noncommutative geometry", JHEP 9909, 032 (1999), hepth/9908142.

A.A. Tseytlin, "Born-Infeld action, supersymmetry and string theory", hep-th/9908105

[6] K.A. Bronnikov and G.N. Shikin, "On the ReissnerNordström problem with a nonlinear electromagnetic field". In: "Classical and Quantum Theory of Gravity", Trudy IF AN BSSR, p. 88, Minsk, 1976 (in Russian)

[7] K.A. Bronnikov, V.N. Melnikov, G.N. Shikin and K.P. Staniukovich, Ann. Phys. (USA) 118, 84 (1979)

[8] E. Ayón-Beato and A. García, Phys. Rev. Lett. 80, 5056 (1998); gr-qc/9911046

[9] E. Ayón-Beato and A. García, Phys. Lett. B 464, 25 (1999); hep-th/9911174

[10] E. Ayón-Beato and A. García, Gen. Relat. E Grav. 31, 629 (1999); gr-qc/9911084

[11] H. Salazar, A. García and J. Plebański, J. Math. Phys. 28, 2171 (1987)

[12] T. Banks, A. Dabholkar, M.R. Douglas and M. O'Loughlin, Phys. Rev. D 45, 3607 (1992);

T. Banks and M. O'Loughlin, Phys. Rev. D 47, 540 (1993)

[13] D. Hochberg and M. Visser, "Geometric structure of the generic static traversable wormhole throat", grqc/9704082.

[14] N. Rosen and H.B. Rosenstock, Phys. Rev. 85, 257 (1952)

[15] Yu.P. Rybakov, "Particle Structure in Nonlinear Field Theory", Moscow, Peoples' Friendship Univ. Press, 1985 (in Russian)

[16] G.W. Gibbons and D.A. Rasheed, Nucl. Phys. B 454, 185 (1995)

[17] M. Novello, V.A. de Lorenci, J.M. Salim and R. Klippert, Phys. Rev. D 61, 045001 (2000); gr-qc/9911085

[18] M. Novello, S.E. Perez Bergliaffa and J.M. Salim, grqc/0003052

[19] P.A.M. Dirac, Proc. R. Soc. London 209A, 291 (1951) 